mean $=6.3+/-1.8 \mathrm{pg} / \mathrm{ml}, p=0.25)$. After TNF $\alpha$ stimulation, protein level of NT3 was significantly higher in CRPS skin fibroblasts (CRPS mean $=10.6+/-2.4 \mathrm{pg} /$ $\mathrm{ml}, \mathrm{HC}$ mean $=4.8+/-1.3 \mathrm{pg} / \mathrm{ml}, p=0.004$ ).

Conclusion: These data indicate a new role of skin fibroblasts in CRPS. Differential basal and stimulated expression of NT3, the receptor for NT3 (TrkC) and NGFR, the common receptor for all neurotrophins, indicates deregulated communication of fibroblasts with the sensory nerve fibers in CRPS. This might contribute to the dysregulated healing process and sustained pain.

Disclosure of Interests: Sanne Stroeve: None declared, Stefan Dudli: None declared, Isabel Kolm: None declared, Irina Heggli: None declared, Nick Herger: None declared, Sabrina Catanzaro: None declared, Andreas Schweizer: None declared, Maurizio Calcagni Speakers bureau: Arthrex, Consultant of: Medartis, Arthrex, SilkBiomaterials, Grant/research support from: Medartis, Oliver Distler: None declared, Florian Brunner: None declared, Astrid Juengel: None declared DOI: 10.1136/annrheumdis-2021-eular.830

\section{OP0086 GENDER INFLUENCE ON CLINICAL MANIFESTATIONS, DEPRESSIVE SYMPTOMS AND BRAIN-DERIVED NEUROTROPHIC FACTOR (BDNF) SERUM LEVELS IN PATIENTS AFFECTED BY FIBROMYALGIA}

C. Gioia ${ }^{1}$, B. Lucchino ${ }^{1}$, C. Iannuccelli', G. Dolcini ${ }^{1}$, M. DI Franco ${ }^{1}{ }^{1}$ Sapienza Università di Roma, Reumatologia, Dipartimento di Scienze Cliniche, Internistiche, Anestesiologiche e Cardiovascolari, Sapienza Università di Roma, Roma, Italy

Background: Fibromyalgia (FM) is a common rheumatic disease characterized by chronic widespread pain, sleep and mood disorders. A higher prevalence of FM in women compared with men is well known, although the specific differences in clinical manifestations related to gender are still poorly defined. Brain-Derived Neurotrophic Factor (BDNF) is an endogenous growth factor that gained attention for its potential as biomarker of several diseases, including FM and depression.

Objectives: The aims of this study were to investigate gender-related difference among males and females affected by FM in clinical manifestations, depressive features and BDNF serum level, evaluating also the diagnostic potential of the latter.

Methods: We consecutively enrolled adult patients affected by FM (ACR 2016) referring to our out-patient clinic. Each subject underwent clinical and answered to questionnaires for the severity of FM symptoms (Revised Fibromyalgia Impact Questionnaire, R-FIQ) and depressive symptoms (Beck Depression Inventory-II, BDI-II). We collected blood samples from a subgroup of patients of both sexes, matched for age, for BDNF serum level dosage through ELISA. BDNF levels were assessed also in a control group, matched for sex and age.

Results: The cohort was composed by $201 \mathrm{FM}$ patients (172 F, 29M), mean age 49.13. Females showed higher values of R-FIQ total score $(p=0,0005)$ as well the specific items of the R-FIQ for pain $(p=0,013)$, fatigue $(p=0,014)$, memory problems $(p=0,007)$, tenderness to touch $(p<0,0001)$, balance problems $(p<0,0001)$ and sensitivity to environmental stimuli $(p=0,012)$ when compared with males (fig. 1). There was no difference in BDI-II between males and females, but notably male patients reported a significantly higher frequency of coexisting depressive disorder ( $p=0,038)$ (fig. 2). Serum BDNF levels were evaluated in $40 \mathrm{FM}$ patients and 40 healthy controls (HC) (F:M 1:1). BDNF levels were significantly lower in FM patients compared with $H C(p<0,0001)$. Among FM patients, BDNF levels were lower in males compared with females $(p<0,0001)$ (fig.3). BDNF did not correlate with any clinical and clinimetric parameter. BDNF showed a good diagnostic performance ( $A \cup C=0,89$, $\mathrm{Cl} 95 \%=0,82-0,9630, \mathrm{p}<0,0001$ ) (fig. 4). At a cut-off value $<6,47 \mathrm{ng} / \mathrm{dl}$, BDNF showed a specificity of $75 \%$ and a sensibility of $92,31 \%,(\mathrm{Cl} 95 \%=79,68-97.35)$ for FM identification ( $\mathrm{LR}=3,692)$.

Conclusion: FM clinical manifestations are strongly dependant from gender. While females present a more severe disease and a higher burden of symptoms, mood disorders tend to be a major characteristic of males with FM. Reduced BDNF serum levels have been reported as typical of depressive disorders. Our findings of lower BDNF levels in male FM patients compared to females support this hypothesis. BDNF have potential as biomarker of the disease and should be validated in larger cohorts.

REFERENCES:

[1] Sarzi-Puttini et al. Nature Reviews 2020

[2] Colucci-D'Amato et al. Int J Molecular Sciences 2020

[3] Nugraha et al. Rheumatol Int 2012
[4] Schmitt et al. Ann Med 2016

[5] Melchior et al. Neuroscience 2016

[6] Stefani et al. Neuroscience Letters 2012
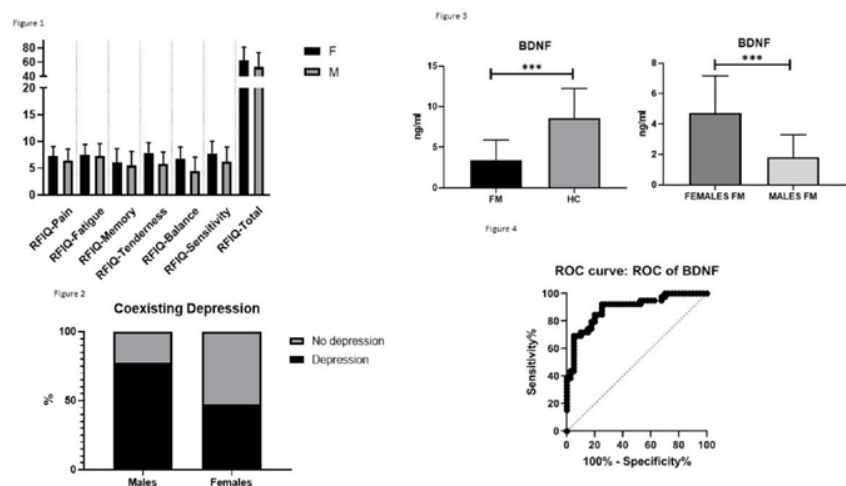

Disclosure of Interests: None declared DOI: 10.1136/annrheumdis-2021-eular.3326

\section{\begin{tabular}{|l|l}
\hline OP0087 COMORBIDITIES IN HAND OSTEOARTHRITIS \\
\hline
\end{tabular} PATIENTS: PREVALENCE AND IMPACT ON PAIN AND PAIN SENSITIZATION}

E. Mulrooney ${ }^{1}$, T. Neogi ${ }^{2}$, H. Solveig Dagfinrud ${ }^{1,3}$, H. Berner Hammer ${ }^{1,4}$, P. Steen Pettersen ${ }^{1}$, T. L. Gaarden ${ }^{5,6}$, K. Engedal ${ }^{7,8}$, T. K. Kvien ${ }^{1,9}$ K. Magnusson ${ }^{10}$, I. K. Haugen ${ }^{1} .^{1}$ Diakonhjemmet Hospital, Division of Rheumatology and Research, Oslo, Norway; ${ }^{2}$ Boston University School of Medicine, Section of Rheumatology, Boston, United States of America; ${ }^{3}$ University of Oslo, Department for Interdisciplinary Health Sciences, Oslo, Norway; ${ }^{4}$ University of Oslo, Department of Orthopaedics, Oslo, Norway; ${ }^{5}$ Diakonhjemmet sykehus, Alderspsykiatrisk Avdeling, Oslo, Norway; ${ }^{6}$ University of Oslo, Faculty of Medicine, Oslo, Norway;

${ }^{7}$ Norwegian Centre of Ageing and Health, Norwegian Centre of Ageing and Health, Oslo, Norway; ${ }^{8}$ University of Oslo, Department of Geriatric Medicine, Oslo, Norway; ${ }^{9}$ University of Oslo, Division of Orthopaedic Surgery, Oslo, Norway; ${ }^{10}$ Lund University, Department of Clinical Sciences, Lund, Sweden

Background: Pain is a hallmark symptom of hand osteoarthritis (OA). A subset of people with hand OA display centrally driven pain characteristics. Pain is driven by person-related factors, but how comorbidities are related to the hand OA pain experience is undetermined. Changes in pain pathophysiology and pain levels by for instants low-grade inflammation, might explain the link.

Objectives: The purpose of this study was to determine whether the burden of comorbidities or the individual comorbidities were associated with pain and pain sensitization in persons with hand OA.

Methods: These cross-sectional analyses included 282 participants with hand OA from the Nor-Hand study. Comorbidities were assessed by an index (subscales: Disease, Limitation and Treatment, (0-45 scale)) by Sangha et al. The participants completed pain questionnaires; Numeric Rating Scale (NRS) hand pain (0-10) and all joints (0-10), Australian/Canadian (AUSCAN) hand pain subscale (0-20 scale), Western Ontario \& McMaster Universities Arthritis Index (WOMAC) pain subscale (0-20) and homunculus (painful joint sites last six weeks, (0-18)). Pressure pain threshold (PPT, handheld algometer, $\mathrm{kg} / \mathrm{cm}^{2}$ ) at tibialis anterior and trapezius and Temporal summation (TS) at the distal radioulnar joint tapped with weighted probe $(x 10)$, assessed central pain sensitization. TS-delta was calculated of the first and peak measure of the fifth or tenth tap. Linear regression was used to examine all relations, while adjusted for age, sex, body mass index and education.

Results: The participants (89\% women, median (IQR) age: 61 (57-66)) showed broad range in pain severity. Most frequent comorbidities were back pain $(n=170,60 \%)$, hypertension $(n=85,30 \%)$, stomach ulcer/abdominal disease $(n=62,22 \%)$ and depression $(n=46,16 \%) .281(99.3 \%)$ participants reported $>1$ 